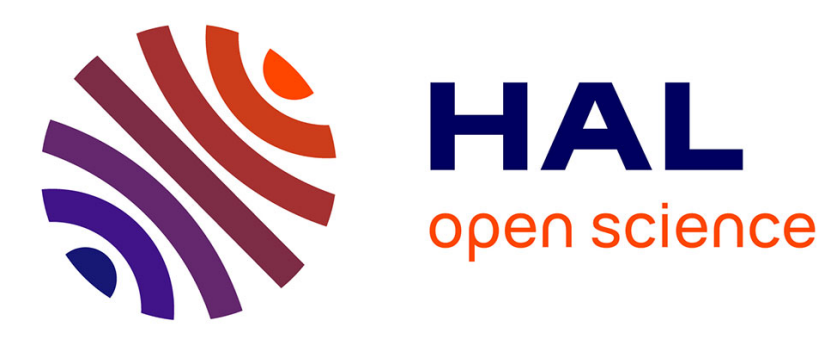

\title{
Effets des structures des réseaux de discussion sur la production des réputations
}

\author{
Alexis Ferrand
}

\section{To cite this version:}

Alexis Ferrand. Effets des structures des réseaux de discussion sur la production des réputations. F.X.Schweyer et G.Cresson. Les usagers du système de soins, Editions de l'ENSP http://www.editions.ensp.fr/sciences-humaines-sociales/recherche-sante-social/152usagers-systeme-soins, pp.313-332, 2000. halshs-00252772

\section{HAL Id: halshs-00252772 \\ https://shs.hal.science/halshs-00252772}

Submitted on 12 Mar 2008

HAL is a multi-disciplinary open access archive for the deposit and dissemination of scientific research documents, whether they are published or not. The documents may come from teaching and research institutions in France or abroad, or from public or private research centers.
L'archive ouverte pluridisciplinaire HAL, est destinée au dépôt et à la diffusion de documents scientifiques de niveau recherche, publiés ou non, émanant des établissements d'enseignement et de recherche français ou étrangers, des laboratoires publics ou privés. 


\title{
Effets des structures des réseaux de discussion sur la production des réputations
}

\author{
Alexis Ferrand, \\ Professeur en sociologie à l'université Lille I, \\ Faculté de sciences économiques et sociales, \\ Institut de sociologie, Membre du CLERSÉ - CNRS
}

\section{Introduction}

L'autorégulation des activités médicales caractérise leur exercice en tant qu'activités professionnelles. Les compétences requises, les diagnostics, les choix thérapeutiques effectués, les réserves qu'impose le code de déontologie, sont en principe contrôlés par la profession ellemême (Freidson, 1970). Cependant, la limitation des ressources économiques a introduit un enjeu financier dans le contrôle des activités médicales. La difficulté rencontrée pour parvenir à endiguer la croissance des dépenses de santé témoigne de la crise d'un des processus de régulation $d u$ « système de santé ${ }^{1}{ }$. L'approche politico-administrative considère que cette régulation économique du système doit mettre en jeu la définition d'objectifs globaux, un autocontrôle des prestataires (individus ou organismes) suivi de sanctions ex-post imposées par une autorité globale. Dans cette approche, les professionnels, les organismes payeurs, et les pouvoirs politiques sont les acteurs essentiels de la régulation économique du système. Aux usagers, on demandera au mieux d'être des demandeurs de soins moins déraisonnables et de veiller à ne pas être inutilement malades. Les échecs répétés des efforts et des plans engagés dans cet esprit sont dus au

1. Cette contribution s'inscrit dans le cadre d'une recherche sur « Les systèmes locaux de santé » (responsable scientifique G. Cresson), soutenue par le programme CNRS «Santé et Société », 1999-2000. 
fait qu'ils laissent entières les contradictions propres à d'autres niveaux de régulation du système de santé (Johanet, 1999).

Mais on peut se demander si ces échecs ne tiennent pas aussi en partie au caractère exclusivement « top down » des contrôles mis en œuvre. Existe-t-il d'autres logiques de régulation déjà à l'œuvre? Évidemment oui, celles inhérentes à la dynamique des rapports offre/demande de soins, à la " consommation » des biens de santé. Nous envisageons ici une forme de régulation «par le bas » du système de santé. Nous partons des "gens », en les considérant comme acteurs sociaux dans le système de santé, lorsqu'ils jouent les rôles spécialisés de patient, usager, consommateur de soin, malade, sans les séparer des rôles généraux (ouvrier, mère, voisin...) qu'ils assument régulièrement. Le modèle normatif du système « libéral » d'accès aux soins suppose le choix « libre » par le patient de son soignant au sein d'une palette d'offres concurrentes. Nous dirons que le fait qu'un acteur envisage de consulter ou de continuer à consulter un soignant relève d'un modèle d'action rationnelle complexe ${ }^{2}$. Mais le patient est dans une situation d'information très imparfaite: que saitil des qualités des soignants et comment peut-il les juger? C'est la circulation d'informations et la production de jugements sur les offres de soins au sein de réseaux de discussion entre acteurs, c'est-à-dire les processus interindividuels de production des réputations, que nous cherchons à formuler dans ce texte. Pour cela, nous devons examiner deux questions:

- d'une part, quelles sont les conditions d'existence de relations de discussion entre les usagers sur les offres de soins?

- d'autre part, comment ces discussions permettent-elles des jugements sur ces offres?

On propose un modèle où la réputation n'est pas une information qui circule dans des canaux, mais le produit toujours redéfini de confrontations d'opinions que réalisent les discussions situées dans des structures relationnelles variées.

L'analyse des relations de discussion articule deux niveaux de conditionnement:

- Le conditionnement normatif. Des normes indiquent dans quel type de relation, entretenue avec quel type de partenaire, on peut discuter telle question, ou indiquent au contraire qu'on ne doit pas discuter telle question.

2. Nous n'ignorons pas que contrôle n'est pas synonyme de régulation, ni que « rôle" et «action" sont des concepts que les manuels n'aiment pas rapprocher. Et nous ne définissons pas ici le «système ». Ce texte ne peut aborder que certains aspects de la problématique. 
- Le conditionnement sociométrique ou structural résultant de la position de cette relation dans un réseau englobant: la " position » de la relation implique qu'elle dépend des autres relations qu'entretiennent les partenaires qui offrent des opportunités de recevoir certaines informations ou bien, au contraire, qui exercent des pressions interdisant certains échanges. Les réseaux présentent des régularités plus ou moins fortes dans l'organisation sociométrique des relations. Les structures sociométriques ne sont pas mécaniquement déductibles des normes ${ }^{3}$. On appellera ici « modèles sociométriques » ou «structures » ces formes d'organisation.

Enfin, nous n'abordons ici qu'un seul des quatre types d'effets des réseaux de discussion:

- Ils assurent la production des cadres cognitifs et normatifs (frames) à partir desquels les individus interprètent les situations. En ce sens, ils encadrent la production des contenus des représentations sociales des acteurs sur leur propre état de santé, les soins, les relations soignant-soigné, etc.

- Ils assurent l'interprétation, la certification et la validation " pour nous » des contenus des messages des médias.

- De manière plus spécifique, les réseaux de discussion assurent la circulation d'informations sur l'offre de soins. Ils permettent l'échange informel plus ou moins développé d'informations entre soignants et habitants.

- Enfin ils assurent la production des évaluations sur les offres, et la stabilisation des notoriétés.

\section{Les normes de discussions sur la santé et les soins}

Les réputations des soignants se font et se défont vraisemblablement dans des discussions qui peuvent aller des confidences dramatiques aux ragots superficiels. Sans avoir examiné systématiquement la littérature, nous supposons que les discussions sur les soignants sont rarement séparées des discussions sur la relation soignant-soigné, qui impliquent de parler aussi de sa propre santé ou de sa maladie. Nous pouvons concevoir que la réputation d'un soignant est un

3. L'idée de différents types « d'ordres » relationnels est banale: on sait bien que certaines relations sont régulées par des normes impersonnelles, et d'autres en fonctions d'informations très personnelles des acteurs les uns sur les autres. Nous mettons l'accent ici sur l'opposition entre contrainte normative et contrainte sociométrique ou triadique: une relation dépend parfois plus de ce que des tiers disent des partenaires que de ce qu'ils savent l'un de l'autre. On trouve dans Clyde Mitchell 1973, p. 2 une formulation liminaire de ce problème. 
jugement sur la manière dont il est susceptible de prendre en charge nos maladies, nous-mêmes comme malades, et que juger un soignant implique de s'impliquer, de parler de sa propre santé. Dès lors, ce sont les conditions normatives de discussion aussi bien sur nos maladies que sur nos soignants qui doivent être examinées.

\section{Maladies stigmatisantes et droit au secret}

Nous identifions ici certaines normes qui indiquent ce qu'un acteur peut dire de sa santé et de ses maladies, en commençant par des normes peu sujettes à variations locales. Il existe par ailleurs des normes soumises à des effets locaux de pouvoir qu'on examinera par après. La maladie est très marquée socialement, et nous identifierons plus facilement des normes qui interdisent/favorisent l'évocation de la maladie, des handicaps, de la mort, que celles relatives à la santé. Peut-on parler de ses propres maux? Est-ce un sujet abordable? Plus en amont, est-ce «bien » d'être malade? Cette question pourrait concerner tout ce que nous savons sur les représentations de la maladie et de la santé. Mais au stade actuel, seuls nous importent les effets normatifs de ces représentations sur la possibilité d'une discussion, et nous nous contenterons de réponses très grossières.

Donc, « est-ce bien d'être malade? » Oui et non, tout dépend non de ce qu'est la maladie, mais de ce qu'elle révèle. Les représentations dominantes des maladies, bien que puissamment cadrées par des interprétations physiologiques positives ${ }^{4}$, articulent parfois désordre organique et désordre social, mal corporel et trouble de l'âme. Si on peut, avec Freidson, considérer que la maladie est toujours une déviance ${ }^{5}$, elle est aussi jugée selon les cas plus ou moins " légitime».

- Il existe des maladies présentables, expressions d'injustes coups du sort, maladies franches et limitées, parenthèses qui affirment la bonne santé habituelle.

- Il existe des maladies "pas bien », punitions vraisemblables de comportements déviants. Si le mal peut être interprété comme conséquence d'une conduite moralement réprouvée (sexualité vagabonde, conduite automobile risquée, toxicomanie...) ou d'une conduite qui

4. «La maladie est considérée comme une entité exogène pénétrée par effraction dans le corps d'un individu qui n'y est pour rien, et la guérison consiste dans la jugulation d'une positivité ennemie. » F. Laplantine (1994), p. 280. Voir également la recherche importante de C. Herzlich (1969).

5. « La maladie est toujours une catégorie de déviation, ou une déviance, par rapport à un ensemble de normes qui représentent la santé ou la normalité », Freidson, (1970, trad. française 1984, p. 213). 
ne respecte pas l'obligation de préserver sa santé (alcoolisme, tabagisme, etc.), il n'est pas bien d'être malade.

- Il existe des maladies « honteuses », car elles concernent des parties du corps « honteuses ».

- Enfin, des maladies chroniques ou évolutives qui entraînent des pertes de capacité et l'image d'une mort annoncée.

Le point important est que si la maladie est de quelque manière honteuse, il existe un relatif droit au secret constitutif de la sphère privée tel que seul l'individu a le pouvoir d'en révéler l'existence à des tiers choisis: il fait des confidences ${ }^{6}$. Si son état concerne la santé publique et le soumet alors à une forme quelconque de contrôle sanitaire, ce droit sera limité, mais non aboli. Les normes de discussions sur la santé et les maladies doivent ainsi être situées à l'intersection de deux domaines:

a) Celui très global des bonnes et mauvaises conduites qui ont des répercussions sur la santé;

b) Celui qui reconnaît un droit à l'autonomie de l'individu, dans sa vie privée, mais soumet ce droit aux exigences supérieures de l'intérêt collectif (prévention et contrôle des épidémies). Chacun de ces domaines est crucial dans l'organisation sociale et on voit en quelques mots comment les discussions sur un état de santé personnel, sur la maladie d'un individu, mettent en jeu des propriétés très fondamentales de l'ordre social ${ }^{7}$. Dès lors, pour l'individu, contrôler l'information sur des maux révélateurs et potentiellement stigmatisants constitue un enjeu majeur au service duquel il va déployer les trésors d'ingéniosité tactiques qu'I. Goffman décrit remarquablement. Beaucoup de malades sont "discréditables », mais chacun peut délimiter «ses risques en deux groupes: l'un nombreux, auquel il ne révèle rien, et l'autre, restreint, auquel il dit tout et dont il espère le soutien » (I. Goffman, 1963, p. 116). En conclusion nous pouvons formuler une première hypothèse:

Hypothèse 1: Plus une maladie est potentiellement stigmatisante, plus rares sont les discussions à son propos.

6. Ce droit a notamment trouvé son expression dans l'institution d'une infraction de « violation du secret professionnel ». « Le droit moderne... considère l'être humain à la fois comme citoyen et comme individu; à ce titre il entend protéger l'individu dans sa sphère privée contre les empiètements d'autres individus. Cela explique qu'au début du XIX'siècle les rédacteurs du Code pénal aient entendu protéger les individus contre les indiscrétions des professionnels. Et ce n'est vraiment qu'à partir de cette date que l'on peut être autorisé à dire qu'une disposition juridique, en prévoyant des sanctions contre des professionnels trop bavards, entendit effectivement protéger les personnes privées. » D. Thouvenin, 1982, p. 38

7. Les débats politiques et éthiques relatifs au droit au secret dont devaient bénéficier les citoyens infectés par le VIH ont bien été des « débats de société ». 


\section{Peut-on discuter de son médecin?}

La seconde question générale concerne les discussions sur la relation soignant-soigné: " comment se comporte le professionnel? ", « quel traitement prescrit-il? », « est-il bien »? Des discussions sur ce genre de questions sont-elles tolérées, encouragées, proscrites? Dans quels cas? Nous venons d'envisager que les discussions sur certaines pathologies ont toutes chances d'être inexistantes ou extrêmement limitées. On peut s'attendre, dans ces cas particuliers, à ce qu'il soit aussi délicat de parler de son ou de ses soignants; car il faudrait réussir à dire comment il soigne sans dire ce pour quoi il soigne.

Hypothèse 2: plus une maladie est stigmatisante, plus rares sont les discussions sur les soignants spécialisés dans leur prise en charge.

En dehors de ces cas particuliers, on sait que le code pénal punit la divulgation par un soignant d'informations sur le patient acquises par l'exercice de son art. Le médecin ne doit pas parler à un tiers de son patient ${ }^{8}$. Que dire de la réciproque: le patient peut-il parler à un tiers de son médecin? Juridiquement, rien ne s'y oppose. Cependant, au terme d'une analyse détaillée des jugements portants sur le secret médical $\mathrm{D}$. Thouvenin suggère le caractère totalement paradoxal de l'utilisation de l'article du code pénal relatif à la violation du secret professionnel. Au total c'est quasiment une situation de non-droit qui est crée dans la mesure où c'est au praticien qu'il revient de définir ce qui est secret, et donc ce qui peut être l'objet de sa propre infraction. Par ailleurs il arrive que des droits s'opposent permettant au praticien d'être dans la légalité s'il ne parle pas, et d'être dans la légalité s'il parle. Finalement, Thouvenin montre que ces incohérences renforcent l'autonomie et le pouvoir des médecins, et que le secret ne protège plus le patient, mais le praticien.

Peut-on penser que le patient «moyen » juge aussi que le secret médical n'est pas si sûr que cela? Si oui, cela peut-il dissuader un patient de discuter de son soignant avec un tiers? Il faudrait supposer que le patient ne parle pas de son médecin, et surtout ne le critique pas, par crainte, car il imagine par exemple que, si celui-ci l'apprenait, il pourrait " pour se venger » parler et divulguer des informations potentiellement stigmatisantes. Cela fait un mauvais scénario de film ${ }^{9}$. Par contre, d'autres hypothèses peuvent être avancées.

8. De nombreuses situations l'imposent ou le permettent cependant, comme l'a analysé en détail Cf. D. Thouvenin, 1982, p. 96 sq.

9. Le nombre de «mauvais » films mettant en jeu les pouvoirs et faiblesses, le charme et l'ignominie des médecins, est considérable. Ils témoignent de la puissance des contenus imaginaires prêts à s'investir sur les figures héroïques de ce monde. En cela, des craintes imaginaires aussi alambiquées que celles de notre mauvais scénario ne sont pas à exclure. Mais ce type de réalité - la réalité des productions imaginaires - n'est pas pris en compte dans ce papier. 
Hypothèse 3: La confiance fondée sur le silence du praticien exigerait une sorte de réciprocité. Si le médecin se tait sur son patient, le patient doit se taire sur son médecin.

La compétence médicale est affirmée dans un rapport de domination idéologique tel que les profanes s'interdisent de juger ${ }^{10}$ leur médecin et d'en parler. La soumission à l'autorité du savoir, qu'ont démontrée les célèbres et inquiétantes expériences de S. Milgram ${ }^{11}$, se traduirait ici par une suspension de tout commentaire sur la pratique du médecin.

Hypothèse 4: Plus un patient appartient à un milieu culturellement dominé moins il s'autorise à parler à un tiers de son médecin et à juger sa compétence.

\section{Dans quel type de relation une discussion sur sa santé ou ses médecins est-elle recommandée?}

Nous dirons ici que les types de relations sont différenciés en fonction des types de rôles. Ces rôles sont définis par des interactions attendues de manière systématique (les attributs " fondamentaux » selon $\mathrm{S}$. Nadel ${ }^{12}$ ) ou recommandées. Les différents rôles ont donc des contenus différents (par définition!...). Un des mérites de l'analyse des réseaux personnels est d'avoir montré, pour un même rôle, à côté des contenus « fondamentaux » normativement attendus, l'existence d'une certaine variété d'autres contenus relationnels. Les discussions sur des sujets donnés sont des interactions caractéristiques des rôles. Certains contenus de discussion ne sont possibles (ou sont strictement proscrits) que dans le cadre d'un certain rôle. Les relations peuvent être plus ou moins polyvalentes, comporter un seul ou plusieurs rôles. Ainsi des relations peuvent être le support de discussion sur

10. Comme le souligne L. Karpik (1989), les professions peuvent réaliser un travail soutenu de légitimation macrosociale de leurs conditions de fonctionnement, des droits et des devoirs qui fondent leur autonomie.

11. Des citoyens quelconques, acceptant de participer à des expériences scientifiques sur l'apprentissage et la mémoire, sont capables d'infliger des décharges électriques mortelles à un de leurs semblables, pourvu qu'un scientifique le demande. «L'idée de la science et la reconnaissance de son utilité en tant qu'entreprise sociale légitime fournissent à l'expérience la justification de l'idéologie dominante./. L'homme est enclin à accepter les définitions de l'action fournies par l'autorité légitime... bien que le sujet accomplisse l'action, il permet à l'autorité de décider de sa signification. » S. Milgram (1974), p.178-181.

12. «La notion de rôle suppose la présence d'une série de caractéristiques liées entre elles... dont la totalité constitue l'originalité d'un rôle donné./.. Du point de vue structural, l'attribut est dit fondamental lorsque la norme qui constitue le rôle a une tolérance nulle. » S. Nadel, 1957, p. 52-64. 
des thèmes plus ou moins nombreux et contrastés. Globalement, l'urbanisation et la modernisation conduiraient les relations interpersonnelles à être de plus en plus spécialisées ${ }^{13}$.

Hypothèse 5: Il n'y a normativement que peu de types de relations dans lesquels il est possible de parler de santé.

Cette spécialisation est notamment sensible dans le cas des relations de discussion où il existe des distinctions par domaines (Laumann, E.O. 1973), mais aussi des distinctions fines dans un domaine ${ }^{14}$. Dès lors il convient de ne pas généraliser, et on peut supposer que :

Hypothèse 6: Des normes de rôle assignent de manière préférentielle à des types différents de relations les discussions qui portent sur la santé ou les médecins «en général », et celles qui portent sur la propre santé de l'acteur ${ }^{15}$ et sur ses médecins.

Deux réserves générales doivent être faites. En matière de relations - comme dans tous les domaines - les prescriptions normatives sont préférentielles et peuvent comporter des clauses conditionnelles: si aucune relation du type préférentiel n'est disponible, alors on peut se « rabattre » sur un autre type de relation. Deuxièmement, s'agissant ici de contrôle de l'information sur soi, les caractéristiques normatives des relations sont renforcées/contrariées par les caractéristiques sociométriques du réseau qui garantit plus ou moins à l'acteur un contrôle de l'information sur lui même. Ces propriétés sociométriques seront évoquées plus loin.

13. Dans le domaine des échanges d'aide B. Wellman conclut (1999, p. 24) "Community ties are narrow specialized relationships, not broadly supportive ties ». Mais, sur la base d'un vaste échantillon stratifié selon la taille des agglomérations, C.S. Fischer (1982, p. 142-143) arrive à des conclusions plus complexes (NB multistrand signifie qu'une relation comporte plusieurs types de liens, ou plusieurs types de contenus échangés) « ...descriptively, urban residents have more multistranded networks, but living in urban places itself neither increase nor decrease strandedness (...) People in the modern sector of society - the young, educated, and urban - have... roughly as multistranded networks as their counterparts in the traditional sector ».

14. Dans le domaine des confidences affectives et sexuelles, les relations où on parle des sentiments ne sont fréquemment pas les mêmes que celles où on parle de maladies sexuelles ou de contraception (Ferrand, 1991). De même dans le domaine professionnel, « certaines relations permettent de parler profession, mais parler seulement, d'autres autorisent également l'éventualité d'un "risque": monter une affaire ou prêter de l'argent » (Ferrand, 1993).

15. Et sans doute aussi selon qu'il s'agit de questions plus ou moins graves... 


\section{Structures sociales locales et domination normative. Effets sur les structures des réseaux de dialogue}

Les normes sociales évoquées jusqu'ici étaient supposées assez générales, globales. On envisage maintenant des caractéristiques variables des «structures sociales locales » qui concerneraient les processus de domination normative. La question est d'identifier des caractéristiques qui modifieraient les régulations normatives dans différents milieux sociaux locaux des discussions sur la santé et des évaluations des offres de soins. Ces caractéristiques concernent la stratification socioprofessionnelle, son expression dans des milieux locaux, et les rapports de pouvoirs entre ces milieux. Trois caractéristiques semblent pouvoir être retenues.

Les hypothèses qui suivent devraient être situées dans un contexte théorique explicite et elles devraient être argumentées sur la base de travaux existants. Ce n'est pas le cas. Disons simplement qu'une recherche personnelle antérieure, et la grande masse de travaux analogues menés simultanément par d'autres collègues, m'ont permis de constater l'existence de processus localisés spécifiques de domination normative ${ }^{16}$.

Hypothèse 7: Si les catégories socioprofessionnelles dont les positions sont définies par des compétences techniques et scientifiques sont particulièrement nombreuses et dominantes, une norme sociale locale de mise en questions de ces savoirs se développe paradoxalement, et la formulation de jugements sur les soignants est plus répandue.

Hypothèse 8: Si les rapports socio-économiques locaux sont marqués par une coupure sociale forte, rendant explicite une opposition entre «bourgeoisie » et « population », l'identification de certains médecins au milieu bourgeois, aux dirigeants, limite les possibilités de jugement critique et/ou le sentiment d'autonomie dans le domaine de la santé des milieux « populaires ».

Les caractéristiques des confrontations politiques locales sont aussi à prendre en compte parce que les processus d'évaluation qui nous intéressent activent des systèmes de valeurs très généraux. Sans pouvoir être plus précis nous supposons que :

Hypothèse 9: Les orientations politiques localement dominantes ont des effets sur les possibilités de parler de la santé et d'exprimer des jugements sur les médecins.

16. Il s'agit d'une monographie sur une banlieue de Grenoble (Meylan) où s'imposaient progressivement les cadres moyens et supérieurs (Cf. Ferrand et al., 1982). Cette recherche faisait partie du programme «Observation du changement social et culturel » du CNRS qui a examiné plus de soixante-dix terrains en France. 
En résumé, l'idée générale est que certaines caractéristiques des structures sociales locales ${ }^{17}$ ont des effets sur des normes locales, qui ont des effets sur les réseaux de discussion sur la santé. L’enjeu essentiel n'est pas le contenu intrinsèque de ces hypothèses, mais leur niveau: il s'agit d'affirmer l'existence de processus locaux et spécifiques d'orientation normative capables d'avoir des effets sur l'établissement de certains types de relations de discussion.

\section{Les conditions relationnelles d'élaboration des évaluations des offres de soins}

Nous allons maintenant aborder un sujet de discussion particulier, celui des « évaluations des offres de soins ». On suppose que ces « évaluations » sont importantes dans le processus par lequel un acteur « choisit » un soignant. On ne discute pas ici le fonctionnement des évaluations comme processus individuels, internes à un acteur. Par contre, nous nous demandons ce qui se passe lorsque nous situons un individu comme terme de différentes relations, et notamment de relations dans lesquelles des évaluations sont mises en discussion de façon plus ou moins explicite? Quelles hypothèses peut-on avancer sur la manière dont ces discussions donnent une forme particulière aux évaluations des acteurs?

\section{Hypothèses sur les évaluations comme confrontations d'opinions}

Trois approches sont mobilisables:

- L'une conçoit l'évaluation comme un processus spécifique à l'individu et les interactions produisent des « influences sur » les processus cognitifs normatifs de l'individu. Le jugement est « dans l'individu ", autrui est une composante de son environnement, une stimulation qui vient de " dehors ". On est proche de la vision courante qui suggère qu'un acteur « $\mathrm{a} »$ des avis, qu'il est propriétaire d'un trésor d'opinions dans lequel il puise pour « exprimer son avis ».

17. Une question classique - à la fois théorique et méthodologique - est également en jeu. L'étude des localités utilise au moins deux niveaux. D’une part, les composantes de la localité (individus, groupes, etc.) peuvent être échantillonnées et analysées, et des généralisations proposées quant aux populations mères respectives dans la localité. D’autre part, à un niveau plus général, la localité elle-même est un « cas » dans une classe de localités. Utiliser dans la construction théorique un référent aussi banal que la composition socioprofessionnelle ou quelques indicateurs de vie politique locale, donne des indications sommaires quant à la classe de localité sur laquelle l'analyse du cas peut vraisemblablement apporter des informations. 
- L'autre conçoit l'évaluation, la formation des opinions, comme des processus collectifs, au niveau des groupes. Le dialogue n'est qu'une actualisation d'un certain état de la " conscience collective». Le jugement serait «dans le groupe».

- Nous retenons ici une troisième vision qui est bien introduite par J. Stoezel (1978, p. 305) lorsqu'il écrit: " On ne sort pas du dilemme: (...) ou c'est l'individu qui produit ses opinions, ou c'est le groupe; ou bien le processus est individuel, ou il est supra individuel. L'hypothèse complètement laissée de côté est celle du processus interindividuel. Or précisément il apparaît de plus en plus que tout un ensemble de phénomènes, dont font partie notamment ceux que l'on conçoit plus ou moins vaguement en parlant d'opinion publique, sont des phénomènes résultant d'interactions entre personnes au sein de groupes primaires. » Nous concevons donc l'évaluation, la formation des opinions dans l'esprit de cette hypothèse " laissée de côté »:

Hypothèse 10: La formation des opinions, les jugements, sont des processus intrinsèques aux communications interpersonnelles. Les opinions sont élaborées dans et par le dialogue. Les interactions ont une capacité de production cognitive et normative, et non simplement de transport ${ }^{18} \mathrm{~d}$ 'une influence ou d'une information qui seraient « traitées » ailleurs, « dans » l'individu.

Le dialogue peut être implicite. En effet, dans une perspective interactionniste, on admet qu'une capacité remarquable de l'esprit est de pouvoir dialoguer avec lui-même comme s'il dialoguait avec un autre. Le dialogue n'est pas exclusivement une situation empirique de discussion entre deux individus qui communiquent physiquement, réellement. Il peut exister comme dialogue silencieux et solitaire « avec un autre soi-même ", ou comme imagination d'un dialogue avec un autre ${ }^{19}$. L'individu comme pensée et jugement ne fonctionne que comme individu en débat (effectif ou potentiel) avec autrui.

Un jugement, une opinion, seraient toujours potentiellement une opinion débattue. Certes, pour juger une relation (de soins) où il est déjà engagé, un acteur s'appuie sur son expérience. Il apprécie de manière solitaire, il se forge une opinion dans son for intérieur. On pourrait dire qu'il a son impression personnelle sur le soin, sans dialogue avec autrui. Mais cette opinion peut éventuellement être sou-

18. On veut insister ici sur le fait que le dialogue reproduit les significations. Ce n'est jamais un simple transfert. Comme l'exprime Dan Sperber (1994, p. 128): "Les représentations tendent à être transformées plutôt qu'exactement reproduites, à chaque fois qu'elles sont transmises... La reproduction exacte d'une représentation mentale par le moyen de la communication est... un cas limite de transformation, la transformation zéro ».

19. «La saisie de rôle est impliquée dans toute communication utilisant des symboles signifiants; cela signifie que l'émetteur imagine - évoque en lui-même comment le récepteur de cette communication la comprend. » A. Rose (1962), p. 9. 
mise à l'épreuve d'une discussion plus ou moins détaillée et sincère avec un proche. Elle devient alors une opinion discutée, interpersonnelle. De manière plus directe, pour évaluer une relation de soin où il n'a pas été engagé, l'acteur doit s'appuyer sur des informations crédibles qui sont le plus souvent fournies par des membres de son réseau personnel («Que penses-tu de la clinique « Gai soleil », on m'a dit que...? »). Ici, l'opinion implique l'intervention d'un tiers et une confrontation d'opinions. Nous supposerons admise l'hypothèse suivante :

Hypothèse 11: Tout jugement, toute opinion, est le résultat d'une discussion, d'un débat avec un tiers. L'absence de débat réel n'est qu'un cas particulier. Donc toute opinion prend la forme d'une confrontation d'opinions.

Ici, les positions théoriques deviennent délicates. Penser que les opinions sont élaborées dans et par le dialogue peut signifier :

- que chaque acteur ne met en jeu, ne reformule, ou ne modifie son opinion que dans une situation d'interaction, étant admis que le reste du temps son opinion, bien à lui, est «stockée » en attente de mobilisation...

- qu'une opinion n'existe que pour le dialogue; que l'acteur n'a telle opinion que pour la donner à et la confronter à celle de telle personne, c'est-à-dire que - sur une seule et même question - il a des opinions relativement à des confrontations et des débats potentiels. En privilégiant cette seconde conception, non d'un acteur miroir, mais d'un acteur qui adapte son opinion à l'échange, on peut former l'hypothèse que...

Hypothèse 12: L'opinion d'un acteur est un ensemble formé de son « opinion », son jugement, PLUS l'argumentaire minimal de soutien de son jugement face à une opinion adverse spécifique ${ }^{20}$.

Dès lors, on peut dire qu'une opinion - comme contenu de pensée - est plus ou moins « ouverte » ou «fermée » en ce qu'elle est plus ou moins prédisposée à telle ou telle nouvelle confrontation. Dire qu'un individu est «borné », c'est dire qu'il a des opinions qui ne sont prédisposées à aucune confrontation. À l'autre extrême, un individu peut « ne plus savoir où il en est » lorsque des opinions d'autrui ont envahi sa propre capacité d'opiner.

On peut dire également qu'une opinion, en tant qu'un jugement et son argumentaire tels qu'ils résultent d'un dialogue, comportent des degrés de consensus variés. Il peut s'agir d'un renforcement de l'opinion préexistante de l'acteur, d'une « convergence communication-

20. Ce n'est pas notre propos immédiat, mais on peut facilement déduire de cette hypothèse la conséquence empiriquement éprouvée qu'un acteur exprime plus facilement «son » opinion lorsqu'il sait que c'est celle de «l'autre », de son interlocuteur. 
nelle », mais le dialogue peut aussi déboucher sur le renforcement d'un désaccord ${ }^{21}$.

Enfin, un acteur a souvent plusieurs partenaires de discussion sur une question, et ils ont souvent des opinions variées. Chacune des personnes avec lesquelles l'acteur discute fait émerger une confrontation d'opinion spécifique. Chaque dyade a un certain effet. La conception que nous proposons fragmente ce que certains supposent être l'opinion homogène et compacte de l'acteur en un certain nombre de variantes adaptées à ces confrontations spécifiques. Que dire alors de ce que juge et pense " globalement » l'acteur sur une question? Simplement, dans le droit fil des propositions précédantes, que :

Hypothèse 13: L'opinion globale d'un acteur sur une question est la combinaison particulière de confrontations d'opinions correspondant à ses discussions avec ses différents partenaires.

\section{Expliquer la dynamique des opinions dans les réseaux}

Dans cette conception, la question de savoir comment « un acteur change d'opinion » doit être posée à deux niveaux différents. À un premier niveau, on dira, c'est une conséquence directe de ce qui précède, qu'un acteur change d'opinion globale lorsque la combinaison des confrontations d'opinions de son réseau de discussion change. Mais comment cette combinaison change? Au niveau de chaque confrontation avec un partenaire spécifique, changer d'opinion c'est soit ne plus discuter avec ce partenaire, soit continuer à discuter avec lui et voir son opinion prendre de plus en plus de place, et s'affaiblir les arguments qui lui étaient opposés.

Dans ce processus, chaque confrontation particulière est-elle influencée par « l'opinion globale » de l'acteur, c'est-à-dire par toutes les autres? La manière de trancher ce point a des conséquences cruciales. Si on dit qu'une confrontation d'opinions répond à une dynamique spécifique, indépendante des autres situations de confrontation de l'acteur, alors il est inutile de proposer une analyse des réseaux d'opinion car nous ne sommes face qu'à des processus dyadiques non connectés. Si on dit qu'une confrontation d'opinion dépend - selon des modalités à préciser - des autres situations de confrontation de l'acteur, alors...

21. «Communication networks consist of interconnected individuals who are linked by patterned flows of information. Such information sharing over time leads the individuals to converge or diverge from each other in their mutual understanding of reality. » E.M. Rogers et D.L. Kincaid (1981), p. 63. 
Hypothèse 14: "L'opinion globale » d'un acteur, c'est-à-dire les opinions qui s'affrontent dans son réseau de discussion conditionnent le contenu et la stabilité de chaque confrontation particulière d'opinion avec un de ses partenaires

Il en résulte qu'une dyade de discussion est conditionnée par les confrontations d'opinions dans les réseaux des deux partenaires, c'està-dire par les deux combinaisons particulières des autres dyades de discussion des deux partenaires. Mais celles-ci sont de même conditionnées par les réseaux des partenaires des partenaires, etc.

Hypothèse 15: Les processus de production et de transformation des opinions fonctionnent dans l'enchaînement des discussions qui se font de proche en proche au sein du réseau. Donc, les transformations d'opinions sont en partie explicables par des propriétés de l'organisation des liens dans des sous ensembles du vaste réseau de dialogue.

Cette proposition soulève une difficulté qu'il faut brièvement évoquer. Le problème est de savoir si les opinions sont dépendantes de la structure sociométrique du réseau ou si la structure du réseau est dépendante des opinions. Dans le premier cas, on dit que les relations ont mille bonnes raisons d'exister telles qu'elles sont indépendamment de l'opinion des gens qu'elles relient, et donc ce sont les structures sociométriques qui vont déterminer comment les opinions vont se diffuser et se modifier. Les relations influencent la dynamique des opinions. Dans le second cas, les relations sont établies entre autres pour les satisfactions qu'elles procurent, notamment celles de rencontrer de préférences des gens qui pensent comme nous, et on cesse de fréquenter ceux qui pensent différemment - éventualité qui vient d'être évoquée plus haut. Les opinions influencent la dynamique des relations. Ce problème n'est pas clairement tranché dans le texte actuel. On peut supposer que des relations interpersonnelles préexistent pour différentes raisons et que l'acteur choisit celles dans lesquelles il va dialoguer. Les réseaux de sociabilité générale constituent une sorte de stock au sein duquel les acteurs sélectionnent pour constituer leurs réseaux spécialisés de discussion sur la santé.

\section{L'exemple de la notoriété: de l'opinion individuelle à la dynamique des opinions dans le réseav englobant}

Pour illustrer ce propos, nous pouvons nous demander ce qu'est la « notoriété » d'un homme politique. Cet exemple est immédiatement parlant et permet d'illustrer certains processus essentiels, même si le choix d'un soignant n'est pas analogue à un vote pour un candidat. Nous allons examiner trois formulations possibles de la notoriété. 
Première formulation, on dit que la notoriété c'est la distribution des opinions des individus. Ici nous avons le modèle bien connu de la popularité des hommes politiques: sur 100 individus 60 pensent que le candidat X est bien pour s'occuper des affaires de la France.

Deuxième formulation, en s'appuyant sur les définitions qui ont été avancées ci-dessus, on dit que la notoriété du candidat c'est la distribution particulières des différentes confrontations d'opinions dans les dyades dialoguantes, c'est-à-dire la distribution des types de combinaisons des opinions positives ou négatives dans les dyades plus ou moins consensuelles. Nous gardons la même distribution des opinions « individuelles » : sur 100 individus, il y en a toujours 60 qui pensent que le candidat $\mathrm{X}$ est bien et 40 qui pensent que $\mathrm{X}$ est mal. Nous allons mettre ces individus en relation les uns avec les autres. Nous supposons que les opinions sont dichotomiques, donc les relations de discussion, les dyades, sont formées par des partenaires dont les opinions respectives peuvent être :

- «bien » \& « bien »,

- «mal » \& «mal»,

- «bien » \& « mal».

Nous supposons ici que tous les individus ont le même nombre de relations de discussion, à savoir une seule: 100 individus forment donc 50 dyades de discussion. Du point de vue des types de confrontations d'opinions que représentent ces dyades, des distributions très différentes sont possibles, que nous illustrons ci-dessous par trois exemples:

\section{Tableau 4: Trois exemples de distributions des types de confrontations d'opinions}

\begin{tabular}{l|r|r|r}
\multicolumn{1}{c|}{ Types de confrontations } & \multicolumn{3}{c}{ Nombre de dyades de chaque type } \\
\multicolumn{1}{c}{ d'opinions dans les dyades } & A & B & C \\
\hline bien-bien & 30 & 20 & 10 \\
bien-mal & 0 & 20 & 40 \\
mal-mal & 20 & 10 & 0 \\
Tous & 50 & 50 & 50 \\
\hline
\end{tabular}

Dans l'exemple A, toutes les relations sont consensuelles, les partenaires des dyades de discussion sont de même avis: les 60 individus qui jugent le candidat X « bien » forment 30 dyades « bien-bien », de même pour les 40 individus qui le jugent « mal », et forment 20 dyades « mal-mal ». Dans l'exemple B les 60 individus jugeant $\mathrm{X}$ «bien » se répartissent entre 20 dyades consensuelles « bien-bien » (soit 40 individus), et 20 dyades dont les partenaires ne sont pas d'accord, type « bien-mal », soit 20 individus d'opinion «bien » et 20 individus d'opinion «mal ». Dans l'exemple C il n'existe plus aucun individu d'opinion « mal » qui soit en relation avec un autre individu d'opinion « mal » : il y a 0 dyade consensuelle « mal-mal ». 
La même distribution des opinions individuelles dans les trois exemples (ce que montrerait un sondage d'opinion classique) est inscrite dans des distributions profondément différentes des dyades de discussion.

Introduisons maintenant une hypothèse très simple sur la dynamique des opinions en supposant qu'un individu qui dialogue avec quelqu'un de même opinion que lui a moins de chances de changer d'avis sous l'effet des campagnes politiques qu'un individu qui dialogue avec quelqu'un d'une opinion différente de la sienne. Alors, pour le candidat X la situation A est parfaitement stable puisque ses 60 partisans forment 30 dyades consensuelles stables: les campagnes adverses ne pourront les affecter, et sa réélection est donc assurée. Mais la situation $\mathrm{C}$ serait très fragile. Parmi ses 60 partisans, 20 forment 10 dyades consensuelles stables, mais 40 sont l'un des partenaires de 40 dyades non consensuelles instables. Le candidat X, qui a pourtant une très large majorité d'avis individuels positifs, peut se trouver en fait face à des risques politiques profondément contrastés.

Une troisième formulation peut enfin être proposée: les discussions n'ont pas lieu seulement dans des relations formant des dyades isolées, mais aussi dans des dyades liées entre elles, c'est-à-dire dans des triades (ou des formes plus complexes). Les types de triades possibles sont:

- «bien-bien-bien $»=\mathrm{BBB}$

- «bien-bien-mal » = BBM

- « bien-mal-mal » = BMM

- « mal-mal-mal » = MMM

Dans une population de 100 individus, décomposés entre 60 qui jugent le candidat $\mathrm{X}$ « bien » et 40 qui le jugent « mal », il y a 33 triades (à un individu près) qui peuvent, se distribuer de manières fort différentes:

\section{Tableau 5: Trois exemples de distributions des types de confrontations d'opinions}

\begin{tabular}{l|r|r|r}
\multicolumn{1}{c|}{$\begin{array}{c}\text { Types de confrontations } \\
\text { d'opinions dans les triades }\end{array}$} & \multicolumn{3}{c}{ Nombre de triades de chaque type } \\
\hline BBB & A & B & C \\
BBM & 20 & 13 & 0 \\
BMM & 0 & 0 & 30 \\
MMM & 0 & 20 & 0 \\
\hline
\end{tabular}

Admettons en arrière-plan la même hypothèse que précédemment sur la dynamique des opinions: un individu qui dialogue avec quelqu'un de même opinion que lui a moins de chances de changer d'avis sous l'effet des campagnes politiques qu'un individu qui dialogue avec quelqu'un d'une opinion différente de la sienne. 
Mais, étant maintenant dans le domaine des triades, chaque individu est confronté à deux partenaires de discussion. Il faut donc complexifier un peu l'hypothèse:

- un individu qui dialogue avec deux partenaires de mêmes opinions que lui n'a aucune chance de changer d'opinion sous l'effet des campagnes ;

- un individu qui dialogue avec un partenaire de même opinion que lui et un autre d'opinion différente a moins de chances de changer d'avis sous l'effet des campagnes politiques qu'un individu qui dialogue avec deux personnes d'opinions différentes de la sienne.

- un individu qui dialogue avec deux personnes d'opinions différentes de la sienne a toutes chances de changer et d'adopter cette opinion contraire.

Cette formulation oblige à regarder les triades du point de vue de chacun de leurs membres, c'est-à-dire à les décomposer en " triplets ", chacun représente un individu et son réseau personnel de discussion. Ainsi, une triade comportant trois personnes 1, 2, 3, et ayant les opinions $\mathrm{BBB}$, est composées de trois triplets analogues, chaque individu a deux dialoguants de même avis que lui. De même pour les triades homogènes MMM. Les triades comportant trois individus 1, 2, 3, ayant les opinions BBM sont décomposables en trois triplets, trois réseaux personnels :

- $1 \mathrm{~B}$ a pour dialoguants $2 \mathrm{~B}$ et $3 \mathrm{M}$

$-2 \mathrm{~B}$ a pour dialoguants $1 \mathrm{~B}$ et $3 \mathrm{M}$

$-3 \mathrm{M}$ a pour dialoguants $1 \mathrm{~B}$ et $2 \mathrm{~B}$

L'individu 3 a toutes chances de changer et d'adopter l'opinion B. La triade devient BBB, elle est alors « équilibrée » et stable. Les modèles BBM et BMM ont des chances d'évoluer par modification d'opinion du minoritaire ou par suppression de la relation. Si on admet ces hypothèses on peut envisager l'évolution de la notoriété de $\mathrm{X}$ selon les trois exemples:

- L'exemple A ne vaut pas la peine que X fasse campagne car il n'y a aucune chance que ses partisans changent d'opinion dans la mesure où ils sont tous membres de triades équilibrées. Avec ses $60 \%$ de partisans, sa réélection est assurée.

- L'exemple B mérite que le candidat X investisse un maximum pour tenter de ne pas perdre les 20 partisans $\mathrm{B}$ qui sont membres des 20 triades BMM: encadrés chacun par deux M, ils ont toutes chances de devenir M. Cette campagne du désespoir a des chances de mal finir, et le candidat qui avait 60 partisans n'en aura plus que 40, perdant ainsi les élections.

- L'exemple C est plein d'avenir pour le candidat X, car non seulement ses 60 partisans forment au minimum des dyades consensuelles (ils sont tous dans les 30 triades BBM où ils ont un partenaire de même 
avis) et ont donc très peu de chances de changer, mais en plus ces partisans encadrent 30 opposants qui le jugent mal (les mêmes 30 triades $\mathrm{BBM}$ ), de sorte que beaucoup d'entre eux vont devenir des supporters, et la popularité de X devrait croître au-delà des $60 \%$.

\section{Conclusion}

Ces processus pourraient être liés entre eux, et les formes peuvent être plus complexes. L'important est de considérer ici les notoriétés comme des distributions d'opinions dans des structures sociométriques typiques. En d'autres termes, ce ne sont pas les distributions des opinions des acteurs atomisés qui constituent les réputations. Ce sont les distributions des opinions des triades qui constituent les réputations. Pour employer un langage ancien, le réseau est une forme « collective » qui englobe évidemment les individus (c'est la vieille opposition), mais il englobe surtout les dyades. En ce sens, les confrontations d'opinions sont produites dans des interactions dyadiques, mais les notoriétés sont produites par l'interdépendance des dyades dans des triades, et à un échelon supérieur dans les agencements relationnels typiques qu'elles forment. Aussi peut-on penser que les dynamiques des notoriétés sont des processus conditionnés par les types d'agencements relationnels, en d'autres termes par les types de «structures » des réseaux de discussion sur les soins.

«Seules les actions réciproques des individus entrent en jeu et développent une dynamique qui par son ampleur prend une apparence objective et dissimule à chacun sa propre contribution; en fait chacun entraîne aussi bien qu'il est entraîné » (Simmel G., Sociologie et épistémologie.)

\section{Bibliographie}

Ferrand A., Mounier L. (1999), The diversity of personal networks in France; social stratification and relational structures, Wellman B. (dir.), Networks in the global village, Boulder, Westview Press, : 185-224.

Ferrand A., Mounier L. (1998), «L'influence des réseaux de confidence sur les relations sexuelles », in Bajos N., Bozon M., Ferrand A., Giami A., Spira A. (dir.), La sexualité aux temps du sida, Paris, PUF, p. 255-304.

Ferrand A. (1997), La structure des systèmes de relations, L'Année sociologique, $47, \mathrm{n}^{\circ} 1$, p. $37-54$.

Ferrand A. (1993), L'analyse des réseaux personnels, HDR, université de Lille I.

Ferrand A., Mounier L. (1993), L'échange de paroles sur la sexualité: une analyse des relations de confidence. Population, $\mathrm{n}^{\circ}$ 5, p. 1451-1476.

Ferrand A. (1991), "La confidence, le corps, le cœur », Le groupe parental, p. 53-58. 
Ferrand A. (1982), Parents, habitants, citoyens: Meylan, banlieue grenobloise, Paris, CNRS.

Fischer C.S. (1982), To dwell among friends: personal network in town and city. Chicago, University Press.

Freidson E. (1970), Profession of Medicine (traduction française: La profession médicale), Paris, Payot, 1984.

Goffman I. (1963), Stigma, Trd francaise Stigmates, les usages sociaux des handicaps, Paris, Seuil, 1975.

Heider F. (1946), Attitudes and cognitive organization, trad. française in Faucheux C. et Moscovici S. (Eds.), Psychologie sociale théorique et expérimentale, Paris, Mouton, 1971

Herzlich C. (1992 [1969]), Santé et maladie, analyse d'une représentation, 2 édition, Paris, EHESS.

Karpik L. (1989), «L'économie de la qualité », Revue française de sociologie, vol. XXX, p. 187-210.

Laumann E.O. (1973), Bonds of pluralism: the form and substance of urban social networks. New York, John Wiley.

Laplantine F. (1994), Anthropologie des systèmes de représentations de la maladie: de quelques recherches menées dans la France contemporaine réexaminées à la lumière d'une expérience brésilienne, in Jodelet D., Les représentations sociales, Paris, PUF.

Lindenberg S. Frey B.S. (1993), « Alternatives, frames, and relative prices: a broader view of rational choice theory », Acta Sociologica, 36, p. 191-205.

Milgram S. (1974 [1995]), Obedience to authority, an experimental view, traduction française: Soumission à l'autorité, Paris, Calmann-Lévy.

Mitchell J.-C. (1973), « Networks, norms, and institutions », in Boissevain J. Mitchell J.-C. (Eds), Network analysis. Studies in human interaction, Paris, Mouton.

Moscovici S. (1992), in Le courrier du CNRS, Dossier sciences cognitives, $\mathrm{n}^{\circ} 79$, octobre.

Nadel S.F. (1970 [1957]), The theory of social structure, London, Cohen et West, trad. Fse, La théorie de la structure sociale, Paris, Éditions de Minuit.

Sperber D. (1994), « L'étude anthropologique des représentations: problèmes et perspectives », in Jodelet D. Les représentations sociales, Paris, PUF.

Stoezel J. (1978), La psychologie sociale, Paris, Flammarion.

Rogers E.M. Kincaid D.L. (1981), Communication networks, New York, Free Press.

Rose A. M. (1962), « A systematic Summary of Symbolic Interaction Theory », in A. M. Rose (dir.) Human behavior and social processes: an interactionist approach, Londres, Houghton Mifflin.

Thouvenin D. (1982), Le secret médical et l'information du malade Lyon, Presses Universitaires de Lyon.

Wellman B. (1999), « The network community », Wellman B. (dir.), Networks in the global village, Boulder, Westview Press, p. 185-224.

Wellman B. Berkowitz S.D. (dir.) (1988), Social Structures. À Network Approach, Cambridge, Cambridge University Press. 
